\title{
A new algorithm for finite element simulation of wedge osteotomies in voxel models with application to the tibia
}

This article was published in the following Dove Press journal:

Orthopedic Research and Reviews

26 January 2010

Number of times this article has been viewed

\author{
Thomas Pressel' \\ Markus D Schofer' \\ Jörg Meiforth ${ }^{2}$ \\ Markus Lengsfeld' \\ Jan Schmitt' \\ 'Department of Orthopaedics \\ and Rheumatology, University \\ Hospital Marburg, Marburg, Germany; \\ ${ }^{2}$ St. Vincentius Kliniken, Klinik für \\ Orthopädie, Karlsruhe, Germany
}

Correspondence: Thomas Pressel Department of Orthopaedics and Rheumatology, Universitätsklinikum Marburg, Baldingerstraße, 35033 Marburg, Germany $\mathrm{Tel}+49$ 642I 58 6369l Fax +49642I 5867007 Email pressel@med.uni-marburg.de

\begin{abstract}
Wedge osteotomies are used to correct bone deformities or change the forces acting on bones and joints in the human body. Finite element models can be employed to simulate the effect of such operations on the bone or adjacent joints. The automatic generation of voxel models derived from computed tomography data is a common procedure, but the major drawback of the method lies in irregular model surfaces. Therefore, the concept of hybrid models combining voxel and tetrahedron meshes was developed. We present an algorithm to simulate wedge osteotomies in voxel models by adding tetrahedron to brick elements. Applicability of the procedure was tested by performing a parametric study using a tibia model created from computed tomography scans taken in vivo applying individually calculated force conditions. The osteotomy angle largely affected maximum stresses: at 2.5 degrees valgus, the stresses at the medial and lateral tibial plateau were equivalent, while increasing valgus angles reduced medial stresses. The algorithm described here is an improvement of former mesh generation procedures and allows a better representation of the geometry at the osteotomy level. The algorithm can be used for all wedge osteotomies and is not limited to the tibia.
\end{abstract}

Keywords: finite element/osteotomy/voxel/pre-operative planning, simulation, mesh algorithm

\section{Introduction}

Automatically generated three-dimensional (3D) voxel models of human bones for finite element analysis were first described by Keyak and colleagues. ${ }^{1}$ A voxel model consists of a grid of nodes, with equal distances between the nodes in each of the three dimensions resulting in uniform eight node brick elements. The main advantage of voxel modeling is the ability to create the model automatically by analyzing consecutive images generated by computed tomography or magnetic resonance imaging. The main disadvantage is the relatively coarse representation of the model surface particularly in curved segments. Even smoothing algorithms ${ }^{2}$ cannot eliminate these relatively rough model surfaces. This major disadvantage lead to the concept of a hybrid model between brick element parts of the model and freely meshed regions employing tetrahedral elements which are leading to a better representation of curved surfaces. An application of this model was published by Schmitt and colleagues ${ }^{3}$ using a model of the proximal femur for simulating wedge osteotomies. Another approach to improve the surface of voxel models was developed by Grosland and Brown. ${ }^{4}$ They used a semi-automatic refinement algorithm to create a better surface representation. 
Osteotomies are used in orthopedics to correct deformities or modify the forces acting on bones and joints in the human body. A common way of performing such an osteotomy is removing a wedge from the bone, closing the resulting gap and fixating the bone fragments. Until now preoperative planning of these operations has usually been achieved by tracing X-ray films that do not allow for a fully $3 \mathrm{D}$ planning of the surgical intervention.

In this paper, the effects of an osteotomy for an individual patient were simulated preoperatively for two reasons: On the one hand the initial geometric and biomechanical situation is different for every patient, and on the other hand the aim of the operation can be different for each underlying pathology.

Finite element models have been used in medicine for simulation of craniomaxillofacial surgery ${ }^{5,6}$ as well as internal fixation methods in the femur ${ }^{7}$ and tibia, ${ }^{8}$ while other authors investigated vertebral osteotomies. ${ }^{9}$

Osteotomies can be simulated using voxel finite element models generated from imaging data. While an osteotomy could be performed by removing voxels in the plane of the osteotomy, this discontinuity would lead to stress artifacts. We describe a new method to simulate wedge osteotomies on automatically generated voxel models of human bone. To demonstrate the feasibility of this method, a parameter study using a model of a human tibia is presented.

\section{Methods}

\section{Tibia model}

For this study, a 3D model of the proximal tibia was created from computed tomography (CT) images of both knees and proximal tibiae. The CT scan (Siemens Somatom; Siemens, Erlangen, Germany) with a slice thickness of $1 \mathrm{~mm}$ had been performed for a different study which was approved by the local ethics commission, and after informed consent of the patient had been obtained. The voxel model was generated by a custom software programme ${ }^{10}$ generating brick elements from image pixels (Figure 1). For model generation and analysis, the finite element analysis software ANSYS 5.6 (ANSYS Inc, Canonsburg, PA, USA) was run on a double processor PC (Intel Pentium III-500 MHz) with 1 GB RAM. The programme macros for osteotomy and force calculation were written in the Ansys Parametric Design Language (APDL). The initial voxel model consisted of 64905 nodes and 49079 eight-node brick elements. The size of each element was $1.0 \mathrm{~mm}$ by $1.43 \mathrm{~mm}$ by $1.43 \mathrm{~mm}$ resulting in a voxel volume of $2.04 \mathrm{~mm}^{3}$. The material properties of the voxel model were assigned to the elements using a linear

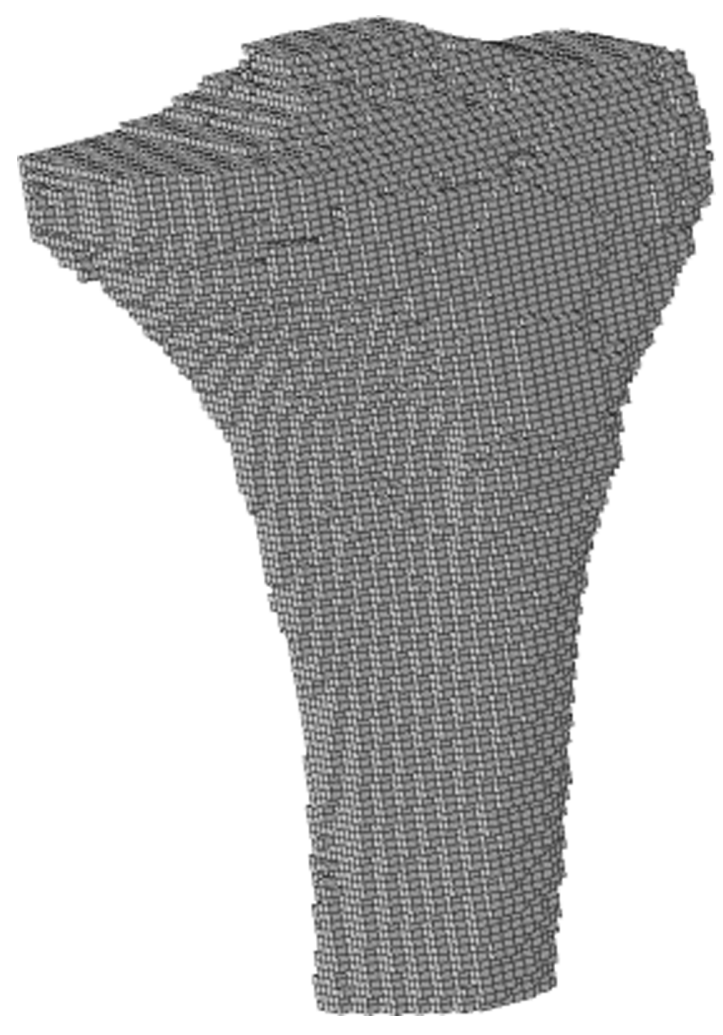

Figure I Front view of the voxel model of the proximal tibia.

relation between Young's modulus (E) and the Hounsfield units (HU) in the CT scan ${ }^{11}$ which quantifies radiographic tissue density. Isotropic material properties were used for all elements.

\section{Osteotomy module}

The osteotomy was simulated in two steps. First, a wedge of elements was defined that had to be removed. This was done by defining two Cartesian coordinate systems that were angled according to the desired osteotomy angle (Figure 2). The first coordinate system was aligned with the proximal osteotomy fragment, while the second coordinate system was oriented along the planned distal osteotomy plane. These local coordinate systems were needed for rotation and translation of the distal osteotomy segment. The elements within the wedge were deleted, separating the model in two parts (Figure 3).

Key points were defined at the positions of the corner nodes that define the interface between the voxels and the newly generated tetrahedron elements. Due to the subsequent rotation and translation of the model, additional key points had to be defined to maintain a rectangular interface between voxel and tetrahedron elements. Elements distal to the osteotomy were selected and rotated and/or translated according to the desired osteotomy result (Figure 4). 


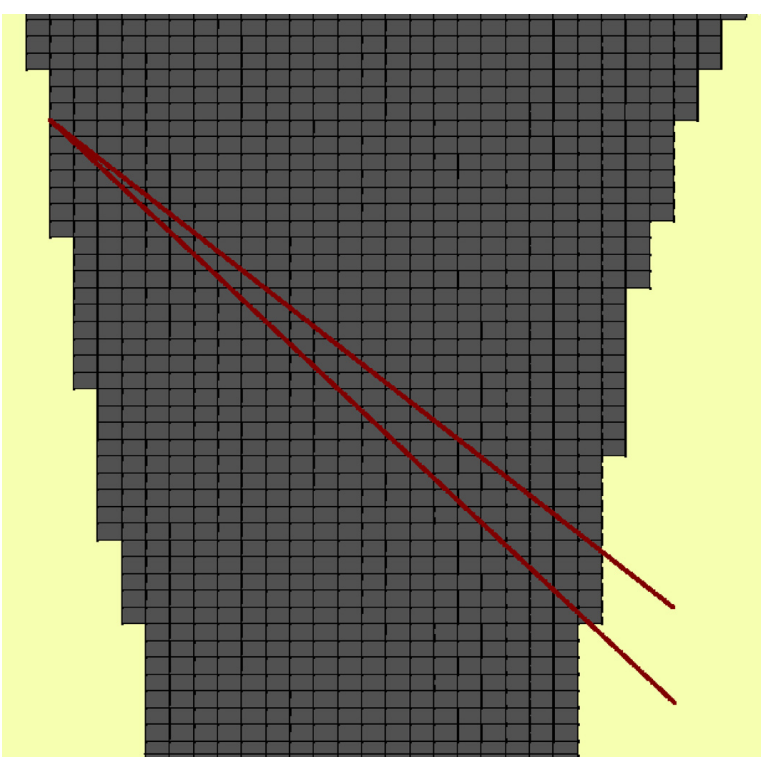

Figure 2 The osteotomy wedge is shown for definition of the voxels that have to be removed (two-dimensional image for better visualization, the model was threedimensional).

In the next step, volumes connecting both osteotomy fragments were created using the predefined key points (Figure 5). The newly created volumes were meshed using tetrahedral elements. The proximal and distal fragments were connected using the node merging option of the ANSYS finite element preprocessor.

\section{Boundary conditions}

The model was constrained at all nodes of the most distal layer. Individual boundary conditions for the proximal

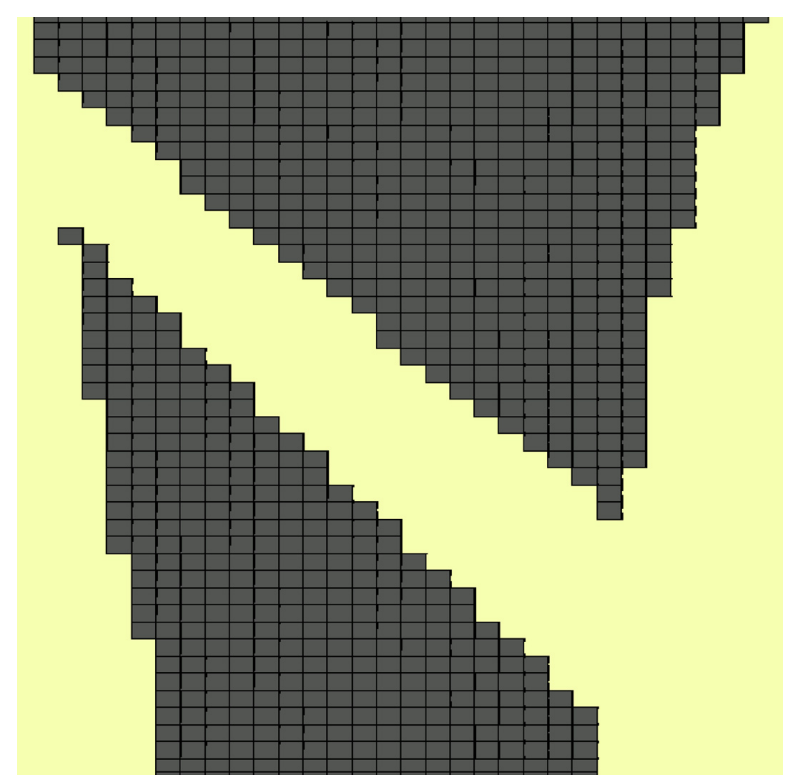

Figure 3 The voxels representing the bone wedge are deleted.

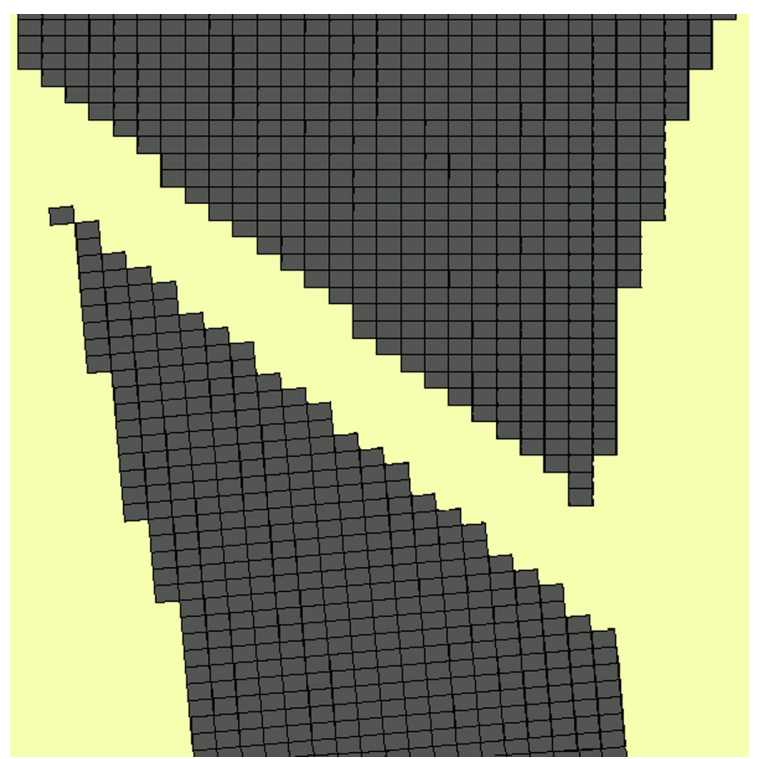

Figure 4 The osteotomy planes are aligned parallel to each other by rotating the distal segment.

femur were defined by two steps: force calculation and force application.

Before the force calculation, the centre of the ankle joint was moved vertically below the centre of a line between both femoral head centers which was approximating the upper body centre of mass. The force calculation module made use of several input parameters to calculate an individual resultant knee joint force and iliotibial tract force in the one leg stance situation based on the two-dimensional models of Maquet. ${ }^{12}$ In this situation, the moments acting at the knee joint are in static equilibrium.

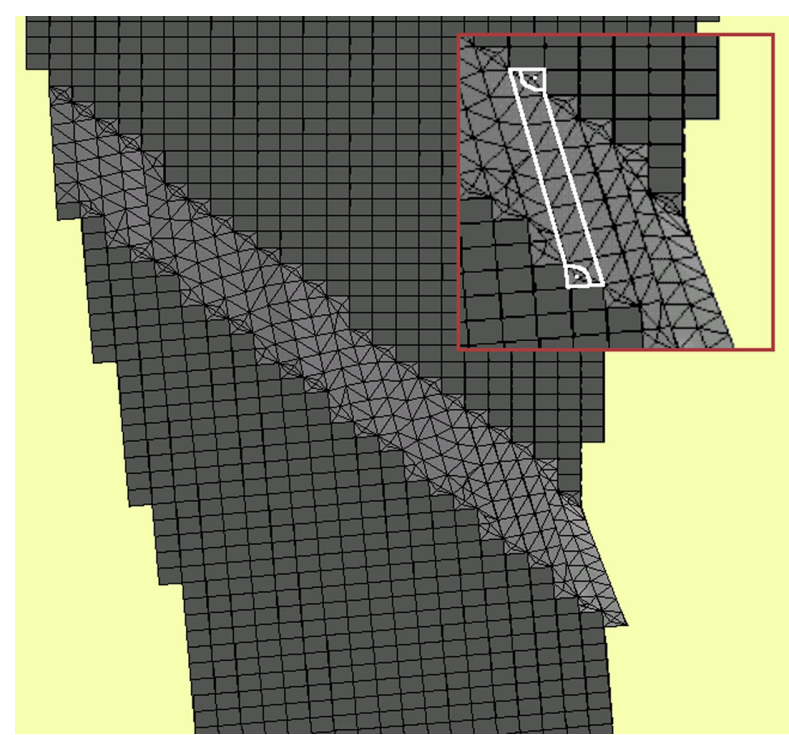

Figure $\mathbf{5}$ The resulting gap is filled by connecting keypoints on the proximal and distal part of the tibia by tetrahedron elements. 


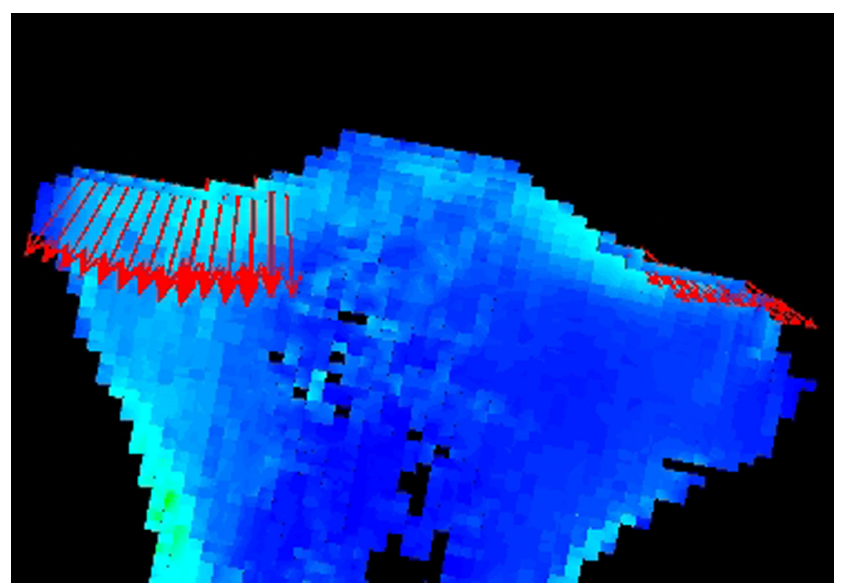

Figure 6 Force vectors acting on the proximal tibia; note that the forces are oriented perpendicular to the joint surface.

For the patient, the initial resultant force magnitude at the tibial head was $1591 \mathrm{~N}$. The distance $(u)$ between the force vector and the centre of the knee joint was $26 \mathrm{~mm}$. In order to consider the change of the biomechanical situation after each osteotomy, the model was realigned with the patient coordinate system, the new coordinates of the knee centre and the ankle joint centre were determined and a recalculation of the force vectors according to the new geometric situation was performed.

The force application macro for the tibial head was programmed to meet three conditions: a) only surface nodes that were in contact with the femoral condyles could be used for force transmission; b) forces could act only normal to the surface of the tibia (Figure 6); c) the force vectors had to add up to the resultant knee joint force vector $(\mathrm{R})$.

Input parameters for the force application macro were the radius of the medial and lateral contact area, magnitude $(R)$ and direction of the resultant knee joint force vector and distance $(u)$ between the knee joint force vector and the centre of the knee.

A new iterative algorithm was used to adjust the magnitude of each individual force vector to meet the above mentioned criteria:

In a first step, all surface nodes that were in contact with the femoral condyles were selected and the normal component was calculated for each node and applied after rotating the local coordinate systems of the node to point to the centre of the medial or lateral condyle. A local cartesian coordinate system was defined at the condyle centre with positive $\mathrm{x}$ directed to the origin of the resultant hip joint force vector. All forces of the nodes in positive $y$ and all forces of the nodes with negative $y$ were added separately, and the forces were adjusted to add up to the resultant hip force and to be of equal magnitude on both sides of the resultant knee force vector. Then the local coordinate system was rotated about the $\mathrm{x}$-axis in 72 steps of five degrees adding up to $360^{\circ}$, and the same adjustment was repeated for each step to balance the force vectors in all three dimensions.

\section{Parametric study}

First the forces for the preoperative model were calculated and applied to the model. Maximum stresses were calculated by the ANSYS solver and stored in the model database. Then a valgus osteotomy was performed on the model. The correction angle was varied from $2^{\circ}$ to $10^{\circ}$ in steps of two degrees. The model was realigned to the one leg stance situation, the new force vector was calculated and applied to the model. Stresses were calculated by the finite element software for each correction angle.

\section{Results}

Results for a varying osteotomy angle are shown in Table 1. In the preoperative situation, the stress in the medial tibial plateau was slightly higher than the lateral stress; the stresses became equivalent when a valgus osteotomy of about $2.5^{\circ}$ was performed. Larger osteotomy wedges lead to higher lateral stresses (Figure 7).

\section{Discussion}

The aim of this study was to provide a tool for the patient specific preoperative simulation of orthopedic osteotomies.

To our knowledge, the work we present here is the first publication of a hybrid model of voxel and tetrahedron meshes for simulation of a tibial osteotomy gap. It combines the advantages of both modeling philosophies by being able to use a fast modeling algorithm while maintaining a smooth interface between the osteotomy fragments. The advantages and disadvantages of voxel-based and geometry-based modeling algorithms have been discussed in previous publications. ${ }^{13,14}$

Table I Influence of different osteotomy angles on the resultant knee joint force vector $(R)$, the distance between the knee joint force vector and the centre of the knee $(\mathrm{u})$ and knee joint stress $(\mathrm{M})$

\begin{tabular}{llll}
\hline Osteotomy angle, $[\alpha]$ & $\mathbf{R}[\mathbf{N}]$ & $\mathbf{u}[\mathrm{mm}]$ & $\mathbf{M}[\mathrm{MPa}]$ \\
\hline Preoperative & 1591 & 26 & \\
$2^{\circ}$ (valgus) & 1593 & 30 & -6.37 \\
$4^{\circ}$ (valgus) & 1595 & 27 & -6.50 \\
$6^{\circ}$ (valgus) & 1597 & 25 & -6.46 \\
$8^{\circ}$ (valgus) & 1599 & 23 & -6.20 \\
$10^{\circ}$ (valgus) & 1600 & 21 & -5.70 \\
\hline
\end{tabular}


Tibial stress

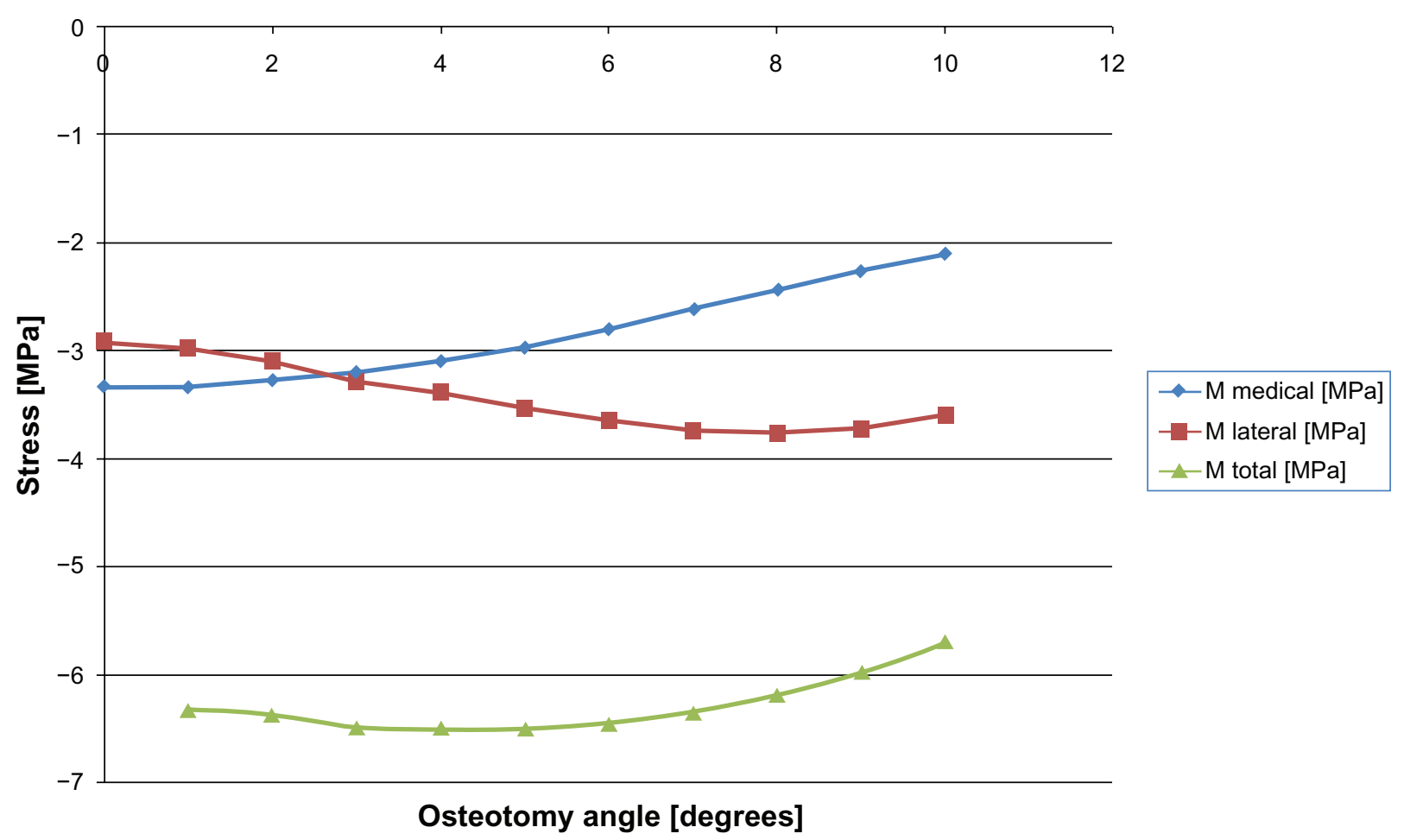

Figure 7 Total stress of the proximal tibia and stresses in the medial and lateral compartment, respectively.

The hybrid modeling appears to be a valuable tool to simulate the biomechanical situation using individual models derived from in vivo $\mathrm{CT}$ data solving the problem of rough interfaces created by voxel models while maintaining the advantages of automatic FE model generation from image data. Since the works of Maquet, ${ }^{12}$ different approaches were used to calculate individual knee joint forces based on geometric features of the patient with the aim of explaining diseases like osteoarthritis or to better understand the effects of operations on the knee joint. Although it is likely that the biomechanical situation in every patient is different and is requiring different diagnostic or treatment methods, a proof for this theory does not exist. The tool presented here allows simulating wedge osteotomies based on individual geometry data and patient-specific hip and knee joint resultant forces. In the future, this tool could be used to simulate the preoperative and postoperative situation in a number of patients with the purpose of answering the question; whether patient specific modeling will yield different individual results.

A possible limitation of the method is the simplification of the boundary conditions at the proximal tibia. Structures like articular cartilage or the menisci were not included in this model, so the stresses at the interface between the femoral and tibial joint surfaces might be lower in vivo.
Although a high stress at the knee joint was found in the one leg stance phase of gait, there are other loading conditions (ie, stair climbing, bicycling, sports activities) that might lead to different values of magnitude and direction of the forces acting on the knee which were not accounted for in this study.

The calculated force vectors are derived from a twodimensional algorithm, therefore a rotation of the force vector in the anteroposterior direction cannot be calculated.

Additional parametric studies on various osteotomy parameters can help to estimate the influence of a combination of parameters for an individual patient.

\section{Conclusion}

This study demonstrates that tibial osteotomies can be simulated by patient-specific 3D finite element analysis. The technique allows simulation of normal and pathologic situations in the human knee joint. To our knowledge, the model presented here is the first 3D model of tibial wedge osteotomy using an automatically created hybrid mesh of voxel and tetrahedron elements.

\section{Acknowledgments}

This work was supported by the Kempkes Foundation in Marburg and German Research Society (LE-1065/1-1). 


\section{Disclosures}

The authors declare that they have no competing interests.

\section{References}

1. Keyak JH, Meagher JM, Skinner HB, Mote CD Jr. Automated threedimensional finite element modeling of bone: a new method. J Biomed Eng. 1990;12:389-397.

2. Camacho DL, Hopper RH, Lin GM, Myers BS. An improved method for finite element mesh generation of geometrically complex structures with application to the skullbase. J Biomech. 1997;30:1067-1070.

3. Schmitt J, Meiforth J, Lengsfeld M. Development of a hybrid finite element model for individual simulation of intertrochanteric osteotomies. Med Eng Phys. 2001;23:529-539.

4. Grosland NM, Brown TD. A voxel-based formulation for contact finite element analysis. Comput Methods Biomech Biomed Engin. 2002;5:21-32.

5. Han UA, Kim Y, Park JU. Three-dimensional finite element analysis of stress distribution and displacement of the maxilla following surgically assisted rapid maxillary expansion. J Craniomaxillofac Surg. 2008;38:145-154.

6. Luboz V, Chabanas M, Swider P, Payan Y. Orbital and maxillofacial computer aided surgery: patient-specific finite element models to predict surgical outcomes. Comput Methods Biomech Biomed Engin. 2005;8:259-265.
7. Chen WP, Tai CL, Shih CH, Hsieh PH, Leou MC, Lee MS. Selection of fixation devices in proximal femur rotational osteotomy: clinical complications and finite element analysis. Clin Biomech (Bristol, Avon). 2004;19:255-262.

8. Blecha LD, Zambelli PY, Ramaniraka NA, Bourban PE, Manson JA, Pioletti DP. How plate positioning impacts the biomechanics of the open wedge tibial osteotomy; a finite element analysis. Comput Methods Biomech Biomed Engin. 2005;8:307-313.

9. Hato T, Kawahara N, Tomita K, et al. Finite-element analysis on closingopening correction osteotomy for angular kyphosis of osteoporotic vertebral fractures. J Orthop Sci. 2007;12:354-360.

10. Schmitt J, Lengsfeld M, Alter P, Leppek R. Use of voxel-oriented femur models for stress analysis. Generation, calculation and validation of CT-based FEM models. Biomed Tech (Berl). 1995;40:175-181.

11. Ciarelli MJ, Goldstein SA, Kuhn JL, Cody DD, Brown MB. Evaluation of orthogonal mechanical properties and density of human trabecular bone from the major metaphyseal regions with materials testing and computed tomography. J Orthop Res. 1991;9:674-682.

12. Maquet P. Biomechanics of the Knee. Berlin, Germany: SpringerVerlag; 1984.

13. Muccini R, Baleani M, Viceconti M. Selection of the best element type in the finite element analysis of hip prostheses. J Med Eng Technol. 2000;24:145-148.

14. Viceconti M, Bellingeri L, Cristofolini L, Toni A. A comparative study on different methods of automatic mesh generation of human femurs. Med Eng Phys. 1998;20:1-10.

\section{Publish your work in this journal}

Orthopedic Research and Reviews is an international, peer-reviewed, open access journal focusing on the patho-physiology of the musculoskeletal system, trauma, surgery and other corrective interventions to restore mobility and function. Advances in new technologies, materials, techniques and pharmacological agents are particularly welcome. The journal welcomes

\section{Dovepress}

original research, clinical studies, reviews \& evaluations, expert opinion and commentary, case reports and extended reports. The manuscript management system is completely online and includes a very quick and fair peer-review system, which is all easy to use. Visit http://www.dovepress. com/testimonials.php to read real quotes from published authors. 